\section{Vers une compréhension du mécanisme de transmission du VIH in utero}

Gaël Vidricaire, Michel J. Tremblay
> La transmission mère-enfant est la première cause d'infection par le virus de l'immunodéficience humaine (VIH) chez l'enfant. Le mécanisme de cette transmission, plus particulièrement au cours de la grossesse, est à ce jour encore mal défini. Les cellules trophoblastiques du placenta sont considérées comme une cible potentielle du rétrovirus ou serviraient éventuellement au passage du VIH vers le fœtus (transcytose). Le processus de transmission du VIH (par infection ou transcytose) serait favorisé, ou encore inhibé, par des facteurs liés à la fois au phénotype viral et à l'environnement cellulaire. <

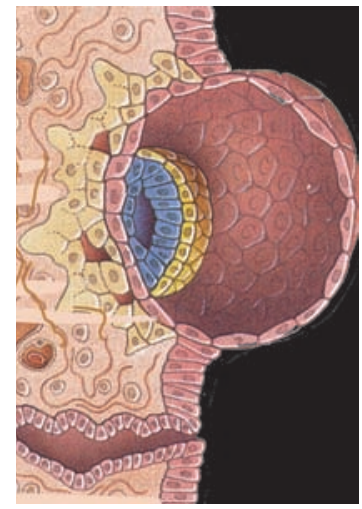

production du facteur
G. Vidricaire, M.J. Tremblay:

Laboratoire

d'immunorétrovirologie

humaine, Centre de recherche en infectiologie, Hôpital CHUL, Centre Hospitalier Universitaire de Québec et Département de biologie médicale, Faculté de Médecine, Université Laval, 2705, boulevard Laurier, Sainte-Foy, Québec, GIV 4G2, Canada. michel.j.tremblay@ crchul.ulaval.ca d'inhibition de la leucémie (LIF) et un polymorphisme HLA mère-enfant réduit [2-4]. Le phénotype viral est aussi à prendre en compte dans la transmission [5].

Le traitement des femmes enceintes par les antiviraux réduit significativement la transmission mère-enfant du VIH: l'administration d'un médicament antirétroviral comme la zidovudine, en trois phases (à partir de la $14^{\mathrm{e}}$ semaine de grossesse, au moment de l'accouchement et durant 6 semaines chez le nourrisson), réduit le taux de transmission initial de $50 \%$ à $68 \%$, pour atteindre un risque de transmission de $8 \%$ [6]. La bithérapie pourrait réduire davantage le risque de transmission qui se situerait alors entre $1,6 \%$ et $4 \%$ [7]; mieux encore, la combinaison thérapeutique HAART (highly active anti retroviral therapy), qui vise à diminuer de façon agressive la charge virale, serait associée à un taux de transmission verticale encore plus faible, de l'ordre de 1\% [6]. Malheureusement, l'accès à ces traitements est fortement limité dans les pays en développement, qui sont pourtant ceux où la majorité des femmes infectées par le VIH vit actuellement. Une meilleure compréhension du moment précis où a lieu la transmission mère-enfant et des mécanismes impliqués est donc requise, ce qui devrait avoir un impact significatif sur le succès du développement de stratégies d'intervention visant à prévenir cette transmission. 


\section{Des périodes critiques pour la transmission mère-enfant}

La transmission verticale du VIH peut se produire au cours de trois stades: en pre-partum (infection en cours de grossesse), où il y aurait passage du VIH de la mère au fœtus via le placenta [8], en intra-partum (infection au cours de l'accouchement) [9] et en post-partum (via l'allaitement maternel) [10]. Deux tiers des enfants contaminés seraient infectés par le VIH le jour même de leur naissance, c'est-à-dire lors de l'accouchement [11]. Au moment de son passage dans le canal utérin, le nouveau-né serait exposé aux sécrétions vaginales et au sang maternel contaminés par le VIH: on observe ainsi une différence d'infection verticale entre le premier (taux plus élevé) et le second des jumeaux nés par voie vaginale [9]; par ailleurs, la probabilité de transmission est réduite de moitié si l'accouchement est réalisé par une césarienne programmée, pratiquée avant le début du travail [9]; enfin, une rupture prolongée des membranes amniotiques lors de l'accouchement favoriserait la transmission du VIH lors du travail et de l'accouchement [12]. La présence du VIH a été détectée dans les sécrétions génitales: il a été postulé que ces virions pourraient accéder à la cavité utérine durant la grossesse, plus particulièrement durant le travail; la transmission mère-enfant périnatale aurait alors lieu par voie ascendante [13].

Qu'en est-il de la transmission mère-enfant durant la grossesse? La contamination in utero, qui concerne un tiers des enfants contaminés, se produirait dans la plupart des cas moins de deux mois avant la naissance [11]; toutefois, elle peut également intervenir durant les premières semaines de grossesse $[8,14]$.

\section{Rôle des trophoblastes}

Au contact de l'endomètre, les trophoblastes entourant le blastocyste se différencient en cytotrophoblastes et en syncitiotrophoblastes; ces der- nières adoptent un phénotype invasif et migrent dans l'endomètre en interagissant avec les cellules déciduales: cela permet l'ancrage et la vascularisation du placenta [15]. Les deux types de cellules forment une bicouche épithéliale polarisée qui baigne dans la circulation maternelle et sépare celle-ci de la circulation fœtale: il s'agit de la barrière placentaire, qui contrôle les échanges materno-fœtaux (Figure 1).

Tout au long de la grossesse, des microlésions entaillent la barrière placentaire, qui est plus mince et vulnérable en fin de grossesse. Il a ainsi été proposé que des cellules infectées, provenant de la circulation maternelle ou de la décidue, ou bien des particules virales libres, puissent directement passer à travers les brèches pour atteindre la circulation sanguine fœtale. Cependant, il semble que la présence de lésions mineures dans la couche de trophoblastes ne soit pas associée à la transmission du VIH, peutêtre grâce à l'intégrité de la membrane basale sousjacente des cellules trophoblastiques ou à l'apparition de cellules fibroïdes dans les lésions [16] (il faut toutefois noter que l'étude ayant donné ces résultats portait sur des femmes ayant une charge virale peu élevée, élément jouant un facteur important dans la transmission). Par ailleurs, si l'inflammation placentaire due à une chorioamniotite aiguë est associée à la transmission du VIH-1, celle-ci survient au moment de la naissance, et non pendant la grossesse [17].

Les autres modèles proposés pour expliquer la transmission du VIH in utero impliquent directement les trophoblastes (Figure 2). Baignant dans la circulation maternelle, ces cellules constituent une cible potentielle pour le VIH: le virus peut-il y entrer? Peut-il s'y répliquer pour atteindre de façon subséquente les cellules fœtales sousjacentes?
A

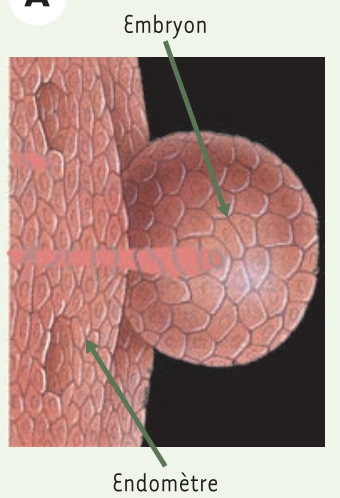

B

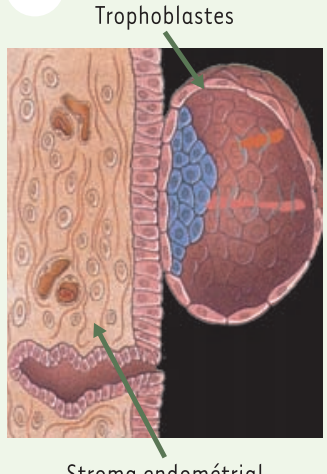

Stroma endométrial

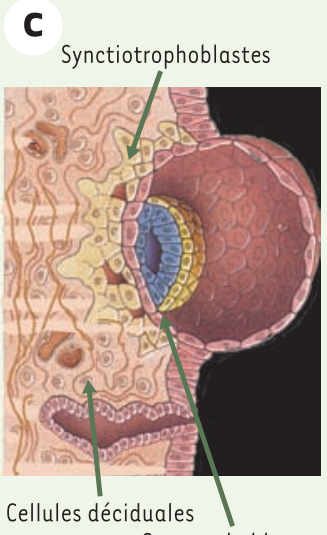

Cytotrophoblastes
Figure 1. Le développement placentaire. A, B. Vues externe et interne de la zone de contact entre l'embryon et l'endomètre. $C$. Les trophoblastes se différencient en deux types de cellules au contact de l'endomètre: les cytotrophoblastes et les syncytiotrophoblastes, qui s'organisent en une bicouche épithéliale polarisée, la barrière placentaire (adapté de [29]).
Si la présence du VIH au sein de trophoblastes, de cellules de Hofbauer et de cellules endothéliales de placentas des premier et troisième trimestres a été suggérée, certains auteurs estiment que seuls les lymphocytes $T$ et les macrophages peuvent être infectés à l'intérieur du placenta. Il est probable que le degré de pureté des préparations de placenta soit à l'origine de cette controverse; par ailleurs, certaines des études ont été menées chez des patientes traitées par des antiviraux, ce qui peut affecter les résultats obtenus [18]. D’une façon générale, les trophoblastes semblent bien susceptibles à l'infection par le VIH, avec toutefois un faible niveau de production virale [19]. Une 
étude phylogénique a cependant montré que les séquences du VIH analysées du côté maternel et du côté des trophoblastes sont reliées, mais avec une évolution des séquences virales au sein des trophoblastes, ce qui implique que le virus est capable d'avoir un cycle complet de réplication au sein des cellules trophoblastiques [20]. L'entrée du virus dans la cellule cible représente une étape cruciale du cycle de vie viral. Rappelons que la pénétration du VIH dans une cellule hôte se fait par fusion entre l'enveloppe virale et la membrane cellulaire. Cet événement se produit après interaction entre le récepteur cellulaire primaire du VIH (la glycoprotéine de surface CD4), un corécepteur cellulaire (un récepteur de chimiokine, généralement CXCR4 ou CCR5) et les protéines gp120 et gp4l de l'enveloppe virale [21]. L'expression de CD4 a été détectée dans les trophoblastes, ce qui accrédite l'idée que l'entrée du VIH dans les trophoblastes pourrait se faire via l'interaction gp120/CD4 [22]. Cependant, d'autres auteurs n'ont pu confirmer l'expression du CD4 dans les trophoblastes, et l'expression des corécepteurs CXCR4 et CCR5 y demeure sujet à controverse [23].

Ce contexte suggérait que, exprimant peu ou pas les récepteurs nécessaires à l'entrée du VIH, les trophoblastes n'étaient guère susceptibles à l'infection par le VIH. En fait, il a récemment été montré que le VIH entrait massivement dans ces cellules, non par fusion avec la membrane, comme c'est le cas pour les lymphocytes T, mais par endocytose; cette variation dans le mode de pénétration du virus a d'importantes conséquences sur la pathogenèse virale, puisque l'endocytose du VIH par les trophoblastes ne paraît pas mener à un cycle productif de réplication [24]. Des auteurs ont rapporté que l'entrée du VIH dans les trophoblastes fait appel à une voie CD4-indépendante; dans cette même étude, la production virale n'était possible que si la souche virale utilisée était exclusivement CD4-indépendante [25]. L'entrée du VIH semble par ailleurs plus importante lorsque les trophoblastes sont en contact avec des lymphocytes $T$ infectés plutôt qu'avec des virions libres. L'adhérence entre les deux types cellulaires formerait une «synapse virale» pouvant favoriser l'endocytose des virions ou l'expression de molécules de surface requises pour l'entrée du VIH [26]. Enfin, l’infection est augmentée in vitro en présence d'anticorps spécifiques du VIH, et réduite en présence d'anticorps dirigés contre les galactosyl céramides, suggérant l'intervention de récepteurs Fc ou de glycolipides dans le processus de pénétration virale [22, 27].

Si le mécanisme d'entrée du VIH dans les trophoblastes et les fonctions des récepteurs cellulaires restent donc sujets à débat, les données accumulées suggèrent qu'une infection des trophoblastes, par suite de leur interaction avec des virus libres ou des cellules maternelles infectées, peut apparaître au cours de la grossesse. De nouveaux virions, bourgeonnant du côté basolatéral, sont ainsi susceptibles d'entrer en contact avec les cellules endothéliales ou les macrophages sous-jacents pour, finalement, atteindre la circulation fœtale.

On sait aussi que les trophoblastes transportent tout au long de la grossesse des macromolécules d'un pôle à l'autre, de la circulation maternelle vers la circulation fœtale: il s'agit du processus de transcytose, utilisé entre autres pour le transfert d'immunoglobulines au fœtus en cours de grossesse. Or le VIH peut être transcytosé du pôle apical au pôle basolatéral des trophoblastes, sans qu'il y ait réplication virale. Ces cellules permettraient donc le passage du VIH non pas via des microlésions, mais directement par le biais de son internalisation par les trophoblastes [27]. C'est au moyen d'un processus similaire que le VIH pourrait traverser les tissus épithéliaux de l'anus ou du vagin lors de la transmission sexuelle.

\section{Rôle de l'environnement placentaire}

Durant toute la grossesse, le placenta et le fœtus expriment une panoplie d'hormones, de facteurs de croissance et de cytokines qui modulent notam-

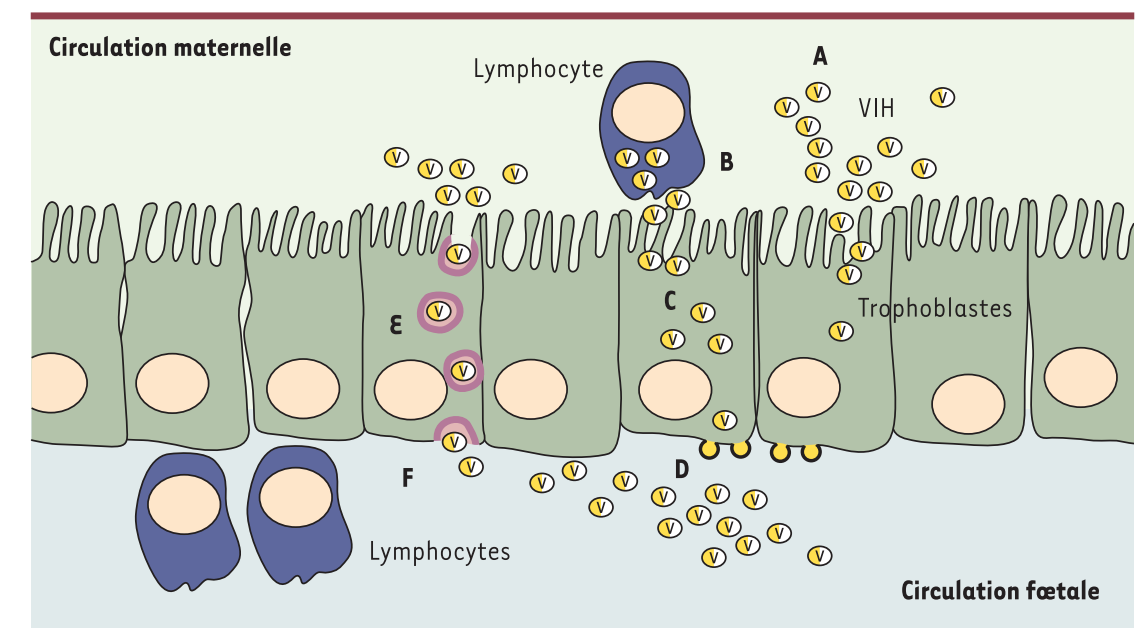

Figure 2. Mécanismes de transmission du VIH in utero. A, B. Des virions libres ou des cellules lymphocytaires infectés (ou des macrophages) présents dans la circulation maternelle viennent au contact des cellules trophoblastiques. C. Le VIH entre alors dans les cellules trophoblastiques, par le biais de mécanismes encore hypothétiques: fusion des virions avec la membrane ou internalisation par endocytose. D. II s'ensuit alors une réplication virale à l'intérieur des cellules trophoblastiques et un bourgeonnement de nouveaux virions au pôle basolatéral (circulation fœtale). $\varepsilon$. Le VIH pourrait également pénétrer dans les trophoblastes par endocytose, et être directement transporté du pôle apical vers le pôle basolatéral sans qu'il y ait infection des cellules trophoblastiques: c'est le processus de transcytose. F. Dans les deux cas (infection des cellules trophoblastiques ou transcytose), le VIH atteint les cellules sous-jacentes, notamment les lymphocytes et les macrophages fœtaux, pour les infecter. 
ment les fonctions placentaires et la croissance des cellules du placenta. $0 r$, les concentrations de TNF- $\alpha$, d'IL- $1 \beta$ et d'IL-6 (interleukines-1 $\beta$ et -6 ) - cytokines exprimées en cours de grossesse - sont plus élevées dans le placenta des femmes infectées par le VIH. De plus, il existe une corrélation positive entre le taux d'expression de TNF- $\alpha$ par les cellules trophoblastiques et le taux de transcription du VIH [28]. Inversement, les femmes qui, infectées par le VIH, n'ont pas transmis le virus à leur nouveau-né, ont une concentration plus élevée de LIF [4]. En conséquence, il semble que les cytokines ou les facteurs de croissance, présents dans l'environnement placentaire et dont l'expression est modulée dans le temps, pourraient jouer un rôle important dans la régulation du cycle de réplication du VIH et la susceptibilité des trophoblastes au VIH. En d'autres termes, selon leur nature, les cytokines pourraient favoriser une infection productive par le VIH ou, au contraire, conférer une certaine protection contre l'infection. Dans cette optique, il est intéressant de noter que les cytokines IL-l et TNF- $\alpha$, activatrices de l'expression virale, sont exprimées par le placenta en début de grossesse puis au moment de l'accouchement, deux stades correspondant précisément aux périodes où les risques de transmission mère-enfant sont les plus élevés [24, 28].

\section{Conclusions}

La transmission verticale du VIH est un processus complexe et multifactoriel. Ce que l'on connaît de la réplication du VIH dans les cellules lymphocytaires semble être difficilement applicable aux cellules trophoblastiques, particulièrement en ce qui concerne le mécanisme d'entrée. De plus, la transmission in utero est fonction de mécanismes de régulation liés à la fois à l'organisation et au développement des cellules trophoblastiques du placenta, aux caractéristiques fonctionnelles et structurales propres aux variants viraux. La combinaison précise d'éléments associés aux facteurs d'origine virale et de l'hôte déterminerait ainsi l'efficacité de la transmission mère-enfant du VIH. La compréhension de l'ensemble des éléments y concourant devrait permettre d'établir de nouvelles stratégies pour contrer la transmission verticale du VIH. $\diamond$

\section{SUMMARY}

For a better understanding of the underlying pathophysiological mechanisms involved in vertical transmission of HIV

Maternal-infant transmission of human immunodeficiency virus-1 (HIV) is the primary cause of this retrovirus infection in neonates. The mechanisms of vertical transmission of HIV, in particular in utero transmission, remain poorly defined. Trophoblastic cells from the placenta are thought to be a target of HIV infection and/or may be utilized by the virus to be transported across the placental barrier by a process known as transcytosis. The vertical transmission of HIV (via infection or transcytosis) may be either favoured or inhibited by factors related to both the viral phenotype and the cellular environment. $\Delta$

\section{RÉFÉRENCES}

1. Onu-sida. Paediatric HIV infection and Aids. Unaids best practice collection. Genève: ONU-SIDA, 2002.

2. Kriebs JM. The global reach of HIV: Preventing mother-to-child transmission. J Perinat Neonatal Nurs 2002; 16: 1-10.

3. MacDonald K, Embree J, Njenga S, et al. Mother-child class I HLA concordance increases perinatal human immunodeficiency virus type 1 transmission. J Infect Dis 1998; 177: 551-6.

4. Patterson BK, Behbahani H, Kabat WJ, et al. Leukemia inhibitory factor inhibits HIV1 replication and is upregulated in placentae from nontransmitting women. J Clin Invest 2001; 107: 287-94.

5. Wolinsky SM, Wike CM, Korber BT, et al. Selective transmission of human immunodeficiency virus type-1 variants from mothers to infants. Science 1992; 255: 1134-37.

6. Cooper ER, Charurat M, Mofenson L, et al. Combination antiretroviral strategies for the treatment of pregnant HIV-1-infected women and prevention of perinatal HIV-1 transmission. J Acquir Immune Def Syndr 2002; 29: 484-94.

7. Mandelbrot L, Landreau-Mascaro A, Rekacewicz C, et al. Lamivudine-zidovudine combination for prevention of maternal-infant transmission of HIV-1. JAMA 2001; 285: 2083-93.

8. Lewis SH, Reynolds-Kohler C, Fox HE, et al. HIV-1 in trophoblastic and villous Hofbauer cells, and haematological precursors in eight-week fetuses. Lancet 1990; 335: 565-8.

9. Goedert JJ, Duliege AM, Amos Cl, et al. High risk of HIV-1 infection for first-born twins. The international registry of HIV-exposed twins. Lancet 1991; 338: 1471-5.

10. Nduati R, John G, Mbori-Ngacha D, et al. Effect of breastfeeding and formula feeding on transmission of HIV-1: A randomized clinical trial. JAMA 2000; 283: 1167-74.

11. Rouzioux C, Costagliola D, Burgard M, et al. Estimated timing of mother-to-child human immunodeficiency virus type l (HIV-l) transmission by use of a Markov model. The HIV infection in newborns french collaborative study group. Am J Epidemiol 1995; 142: 1330-7.

12. Landesman SH, Kalish LA, Burns DN, et al. Obstetrical factors and the transmission of human immunodeficiency virus type 1 from mother to child. The women and infants transmission study. N EnglJ Med 1996; 334: 1617-23.

13. Chuachoowong R, Shaffer N, Siriwasin W, et al. Short-course antenatal zidovudine reduces both cervicovaginal human immunodeficiency virus type I RNA levels and risk of perinatal transmission. Bangkok collaborative perinatal HIV transmission study group. J Infect Dis 2000; 181: 99-106.

14. Laure F, Courgnaud V, Rouzioux C, et al. Detection of HIVI DNA in infants and children by means of the polymerase chain reaction. Lancet $1988 ; 2: 538-41$.

15. Morrish DW, Dakour J, Li H. Functional regulation of human trophoblast differentiation. J Reprod Immunol 1998; 39: 179-95.

16. Burton GJ, O'Shea S, Rostron T, et al. Significance of placental damage in vertical transmission of human immunodeficiency virus. J Med Virol 1996; 50: 237-43.

17. Mwanyumba F, Gaillard P, Inion I, et al. Placental inflammation and perinatal transmission of HIV-1.J Acquir Immune Def Syndr 2002; 29: 262-9.

18. Chandwani S, Greco MA, Mittal K, et al. Pathology and human immunodeficiency virus expression in placentas of seropositive women. J Infect Dis 1991; 163: 1134-8.

19. Mognetti B, Moussa M, Croitoru J, et al. HIV-1 co-receptor expression on trophoblastic cells from early placentas and permissivity to infection by several HIV-1 primary isolates. Clin Exp Immunol 2000; 119: 486-92.

20. Zachar V, Zacharova,V, Fink T, et al. Genetic analysis reveals ongoing HIV type 1 evolution in infected human placental trophoblast. AIDS Res Hum Retrovir 1999; 15: 1673-83.

21. Frankel AD, Young JA. HIV-1: fifteen proteins and an RNA. Annu Rev Biochem 1998; 67: 1-25.

22. David FJ, Tran HC, Serpente N, et al. HIV infection of choriocarcinoma cell lines derived from human placenta: the role of membrane CD4 and Fc-Rs into HIV entry. Virology 1995; 208: 784-8.

23. Mano H, Chermann JC. Fetal human immunodeficiency virus type 1 infection of different organs in the second trimester. AIDS Res Hum Retrovir 1991; 7: 83-8.

24. Vidricaire G, Tardif MR, Tremblay MJ. The low viral production in trophoblastic cells is due to a high endocytic internalization of the human immunodeficiency virus type 1 and can be overcome by the pro-inflammatory cytokines tumor necrosis factor- $\alpha$ and interleukin-1. J Biol Chem 2003; 278: 15832-41.

25. Al-Harthi L, Guilbert LJ, Hoxie JA, et al. Trophoblasts are productively infected by CD4-independent isolate of HIV type 1. AIDS Res Hum Retrovir 2002; 18: $13-7$.

26. Arias RA, Munoz LD, Munoz-Fernandez MA. Transmission of HIV-1 infection between trophoblast placental cells and T-cells take place via an LFA-1-mediated cell-tocell contact. Virology 2003; 307: 266-77.

27. Lagaye S, Derrien M, Menu $\varepsilon$, et al. Cell-to-cell contact results in a selective translocation of maternal human immunodeficiency virus type 1 quasispecies across a trophoblastic barrier by both transcytosis and infection.J Virol 2001; 75: 4780-91.

28. Moussa M, Roques $P$, Fievet $N$, et al. Placental cytokine and chemokine production in HIV-1-infected women: Trophoblast cells show a different pattern compared to cells from HIV-negative women. Clin Exp Immunol 2001; 125: 455-64.

29. Tortora GJ. Development during pregnancy. In: Principles of human anatomy, $9^{\text {th }}$ ed. New York: John Wiley, 1999.

\section{TIRÉS À PART}

M.J. Tremblay 